\title{
Magnetocaloric Effect and Transition Order in $\mathrm{HoAl}_{2}$
}

\author{
S. BARAN ${ }^{a, *}$, R. DURAJ $^{b}$ AND A. SZYTUŁA ${ }^{a}$ \\ ${ }^{a}$ M. Smoluchowski Institute of Physics, Jagiellonian University, S. Łojasiewicza 11, PL-30348 Kraków, Poland \\ ${ }^{b}$ Institute of Physics, Technical University of Cracow, Podchorążych 1, PL-30-084 Kraków, Poland \\ (Received July 18, 2014; revised version November 18, 2014; in final form January 20, 2015)

\begin{abstract}
Magnetic measurements of $\mathrm{HoAl}_{2}$ are reported. The compound crystallizes in the cubic $\mathrm{MgCu}_{2}$-type crystal structure. $\mathrm{HoAl}_{2}$ exhibits two successive magnetic transitions: below $T_{\mathrm{c}}=29 \mathrm{~K}$ a ferromagnetic order is formed while anomaly at $T_{\mathrm{t}}=20 \mathrm{~K}$ is related to reorientation of magnetic moment. Near transition temperatures a magnetocaloric effect with magnetic entropy changes $-\Delta S_{\mathrm{m}}$ equal to $6.3 \mathrm{~J} /(\mathrm{mol} \mathrm{K})$ at $T_{\mathrm{c}}$ and $5.0 \mathrm{~J} /(\mathrm{mol} \mathrm{K})$ at $T_{\mathrm{t}}$ for an external field $\mu_{0} H=9 \mathrm{~T}$ is observed.
\end{abstract}

DOI: $10.12693 /$ APhysPolA.127.815

PACS: 75.30.Cr, 75.30.Kz, 75.30.Sg

\section{Introduction}

The magnetocaloric effect (MCE) is of great interest nowadays [1], especially for its potential application in new generation of refrigerators. The $\mathrm{HoAl}_{2}$ intermetallic compound crystallizes in the $\mathrm{MgCu}_{2}$-type cubic Laves structure (space group $O_{h}^{7}$, respectively $F d 3 m$ ). $\mathrm{HoAl}_{2}$ is a ferromagnet with Curie temperature of $28 \mathrm{~K}$ as reported from specific heat data [2] or $33 \mathrm{~K}$ found from magnetic susceptibility measurements [3]. At about $20 \mathrm{~K}$ easy direction of magnetization changes from $\langle 110\rangle$ to $\langle 100\rangle$ direction [3, 4]. Magnetocaloric effect in $\mathrm{HoAl}_{2}$, determined from magnetic measurements on polycrystalline sample in magnetic field of $5 \mathrm{~T}$, is reported in Refs. [5-7]. The MCE curve has a maximum at $T_{\mathrm{c}}$. The experimental value is smaller than theoretical prediction $6.6 \mathrm{~J} /(\mathrm{mol} \mathrm{K})$ at $H=5 \mathrm{~T}$ [7].

In the present paper we report results of magnetization measurements on bulk $\mathrm{HoAl}_{2}$ sample between 1.9 and $300 \mathrm{~K}$ in magnetic fields up to $9 \mathrm{~T}$. From these measurements we determine temperature variation of magnetic entropy change $\left(-\Delta S_{\mathrm{m}}\right)$ from $M(H, T)$ curves.

\section{Experimental methods and results}

The $\mathrm{HoAl}_{2}$ sample was prepared from $3 \mathrm{~N}$ pure $\mathrm{Ho}$ and $4 \mathrm{~N}$ pure $\mathrm{Al}$. The stoichiometric amounts of the metal were melted in arc under argon atmosphere. Next, the sample was annealed at $1073 \mathrm{~K}$ for one week in evacuated quartz tube. Powder X-ray diffraction measurements performed with $\mathrm{Cu} K_{\alpha}$ radiation indicate that the compound crystallizes in cubic system of the $\mathrm{MgCu}_{2}$-type. The determined lattice parameter equal to $7.838(3) \AA$ is in good agreement with the values reported in literature. Magnetic measurements were performed in temperature range from $1.9 \mathrm{~K}$ to $300 \mathrm{~K}$ and in external magnetic fields up to $9 \mathrm{~T}$ using a vibrating sample magnetometer (VSM) option of the PPMS Quantum Design platform.

* corresponding author; e-mail: stanislaw.baran@uj.edu.pl
The results of magnetic measurements of $\mathrm{HoAl}_{2}$ are shown in Fig. 1. The Curie temperature $T_{\mathrm{c}}=29 \mathrm{~K}$ has been defined as temperature at which $|\mathrm{d} \chi / \mathrm{d} T|$ reaches its maximum for the zero field cooling (ZFC) and field cooling $(\mathrm{FC})$ curves. Below $T_{\mathrm{c}}$ a ferromagnetic order with Ho magnetic moment equal to $7.5 \mu_{\mathrm{B}}$ at $1.9 \mathrm{~K}$ is observed. The hysteresis loop at $1.9 \mathrm{~K}$, presented in lower inset in Fig. 1, reveals presence of a very small coercivity, less than $60 \mathrm{Oe}$, which is favorable for magnetocaloric materials.

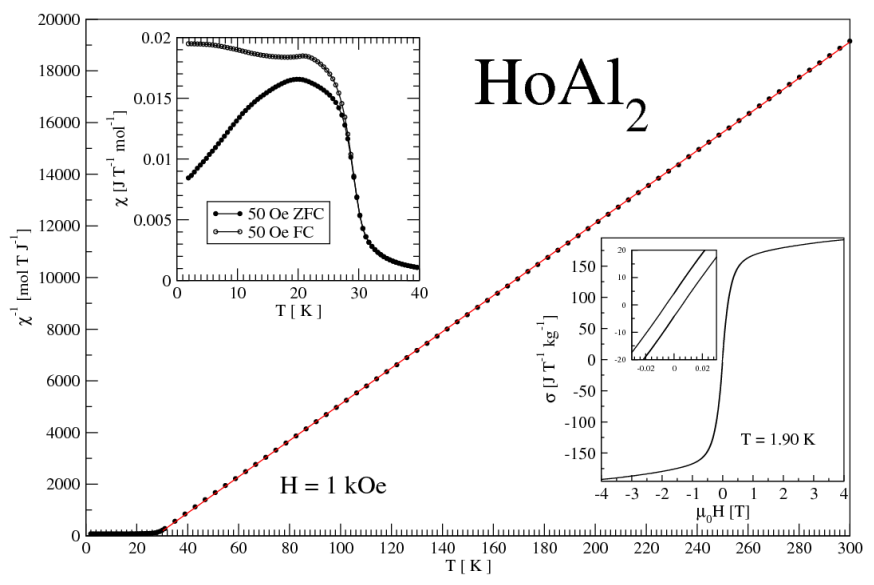

Fig. 1. Temperature dependence of $\mathrm{HoAl}_{2}$ reciprocal magnetic susceptibility. The solid line shows the CurieWeiss law fit. The upper inset presents magnetic susceptibility (ZFC and FC curves) at low temperature while the lower one - hysteresis loop at $1.9 \mathrm{~K}$ with low-field region being enlarged.

Apart from divergence between $\mathrm{ZFC}$ and $\mathrm{FC}$ curves appearing below $T_{\mathrm{c}}$ there is additional anomaly observed in both the curves about $20 \mathrm{~K}$. Above $T_{\mathrm{c}}$ the reciprocal magnetic susceptibility obeys the Curie-Weiss law with paramagnetic Curie temperature equal to $27 \mathrm{~K}$ and effective magnetic moment of $10.7 \mu_{\mathrm{B}}$ which is close to the free $\mathrm{Ho}^{3+}$ ion value $\left(10.61 \mu_{\mathrm{B}}\right)$.

Figure 2a shows magnetization curves measured in applied magnetic field up to $9 \mathrm{~T}$ and temperature ranging from 10 to $40 \mathrm{~K}$ with temperature step $\Delta T=2.5 \mathrm{~K}$. On the basis of these data, isothermal magnetic entropy 
changes were calculated from the relation

$$
\Delta S_{\mathrm{m}}(T)=\sum_{j} \frac{\sigma_{i+1}\left(H_{j}\right)-\sigma_{i}\left(H_{j}\right)}{T_{i+1}-T_{i}}\left(H_{j}-H_{j-1}\right),
$$

where magnetic field $H_{0}=0$ and $H_{j \max }=H$ with $j=1$, $\ldots, j_{\max }$ while $\sigma_{i}$ and $\sigma_{i+1}$ were the values of magnetization at temperature $T_{i}$ and $T_{i+1}$, respectively [8]. Magnetic entropy change vs temperature, for several external magnetic fields, is shown in Fig. 2b. The entropy curves have a maximum at $T=28 \mathrm{~K}$. The maximal entropy change increases with increasing external magnetic field reaching $6.3 \mathrm{~J} /(\mathrm{mol} \mathrm{K})$ at $9 \mathrm{~T}$.
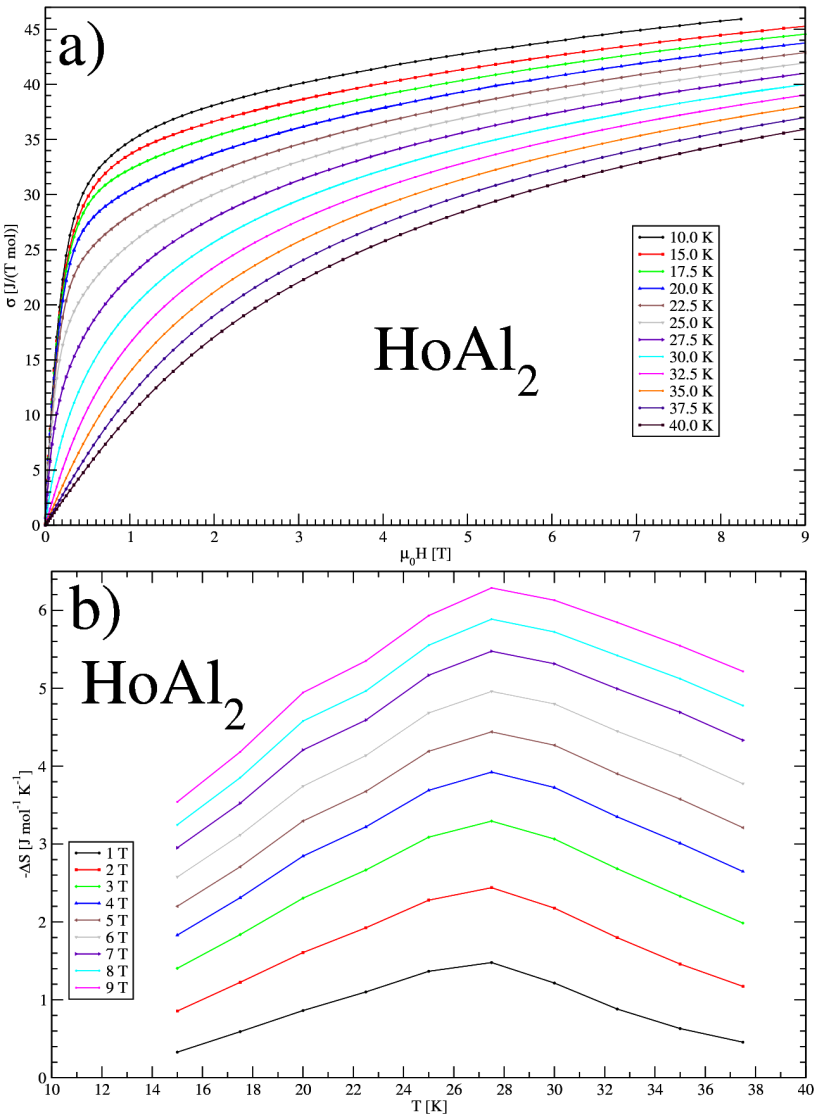

Fig. 2. (a) Magnetization versus magnetic field at different temperatures between 10 and $40 \mathrm{~K}$ and (b) entropy change $-\Delta S$ plotted versus temperature at different magnetic fields up to $9 \mathrm{~T}$.

Refrigerant capacity (RC) can be calculated by the expression $\mathrm{RC}=\Delta S_{\mathrm{m}}\left(T_{\mathrm{h}}-T_{\mathrm{c}}\right)$ where $-\Delta S_{\mathrm{m}}$ is a maximal entropy change at selected field, $T_{\mathrm{c}}$ is a temperature at which entropy change reaches its maximum equal to $-\Delta S_{\mathrm{m}}$ while $T_{\mathrm{h}}$ is a temperature at which $\Delta S$ equals $\frac{1}{2} \Delta S_{\mathrm{m}}[9]$. The refrigerant capacity calculated from experimental data equals about $95 \mathrm{~J} / \mathrm{mol}$ at $9 \mathrm{~T}$.

Magnetic entropy vs. applied magnetic field dependence can be analyzed numerically at temperatures close to $T_{\mathrm{c}}$. Within the molecular field approximation [10], the dependence $\Delta S(T, H) \sim H^{n}$ is predicted. Figure 3 shows

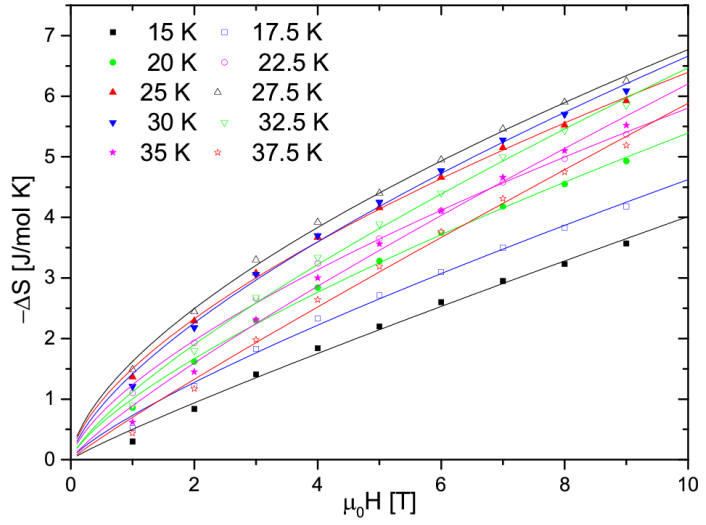

Fig. 3. Magnetic entropy vs. applied magnetic field dependence.

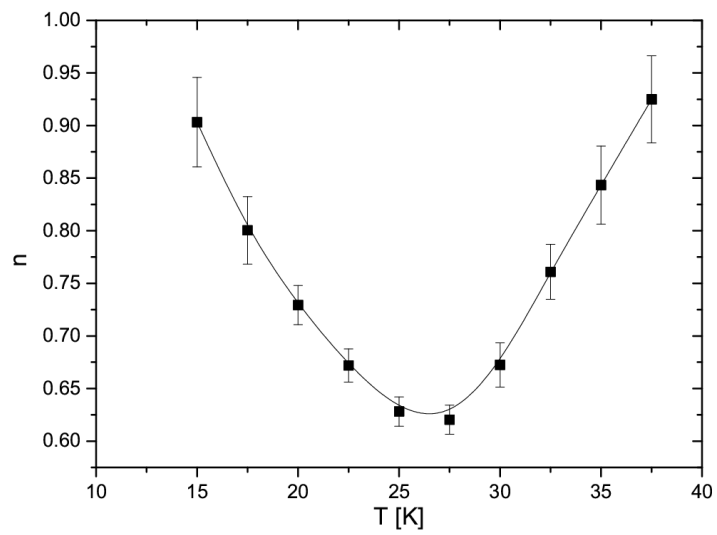

Fig. 4. Temperature dependence of power $n$ determined from relation $\Delta S(T, H) \sim H^{n}$.

both the experimental and fitted data of $\Delta S$ while the obtained values of $n$ are presented in Fig. 4. $n$ changes with temperature reaching a minimum equal to 0.62 at $T=26 \mathrm{~K}$ which is close to the theoretical value of $2 / 3$ (0.667) [10]. While receding from the critical temperature $n$ increases, however, it does not reach either 1.0 or 2.0 as predicted theoretically for low- and hightemperature limit, respectively. Such a result indicates that analyzed temperature range was relatively narrow.

\section{Summary}

In the present work dc magnetization measurements of polycrystalline $\mathrm{HoAl}_{2}$ sample are reported. On basis of experimental data, magnetocaloric effect is investigated. Temperature dependences of magnetization and magnetic susceptibility are in agreement with the previous data assuming that the compound is ferromagnetic with magnetic moments localized on $\mathrm{Ho}^{3+}$ ions.

The new result is a large thermo-magnetic irreversibility between $\mathrm{ZFC}$ and FC curves which is a typical feature associated with a large anisotropy of a ferromagnet. 
The calculated magnetic entropy at $\mu_{0} H=9 \mathrm{~T}$ and $T_{\mathrm{c}}$ equals $6.3 \mathrm{~J} /(\mathrm{mol} \mathrm{K})$ and is comparable with previously reported data $[6,7]$.

Close to $T_{\mathrm{c}}, \Delta S$ behaves as $H^{n}$ thus confirming molecular field approximation predictions. Also the obtained values of $n$ are in agreement with theory.

During the time of our experiment a new paper reporting data on magnetocaloric effect in $\mathrm{HoAl}_{2}$ single crystal was published [11]. The paper reports on temperature dependence of magnetization for $\mathrm{HoAl}_{2}$ single crystal measured along [100] and [110] directions. On the basis of experimental data a thermal variation of magnetic entropy change $\left(-\Delta S_{\mathrm{m}}\right)$ is calculated. Both the $\Delta S_{\mathrm{m}}$ curves, along [100] and [110] directions, have a maximum at $T_{\mathrm{c}}$ and similar dependence above the Curie point. Below $T_{\mathrm{c}}$ a large divergence between the curves is observed: the curve along [110] direction has only small anomaly at $T_{\mathrm{t}}$, similar to that observed in this work, while the curve along [100] direction has a minimum at $T_{\mathrm{t}}$. The value of $-\Delta S_{\mathrm{m}}$ reported in Ref. [11] equals $6.5 \mathrm{~J} /(\mathrm{mol} \mathrm{K})$ at $\mu_{0} H=7 \mathrm{~T}$ and is similar to the one reported in this work. For comparison, specific heat data for polycrystalline sample yield $5.5 \mathrm{~J} /(\mathrm{mol} \mathrm{K})$ at $\mu_{0} H=5 \mathrm{~T}[8]$. Theoretical predictions for MCE in $\mathrm{HoAl}_{2}$ show influence of spin reorientation from [110] to [100] direction on magnetocaloric effect [12].

\section{Acknowledgments}

The research was carried out with the equipment purchased thanks to the financial support of the European Regional Development Fund in the framework of the Polish Innovation Economy Operational Program (contract no. POIG.02.01.00-12-023/08).

\section{References}

[1] O. Tegus, E. Brück, K.H.J. Buschow, F.R. de Boer, Nature 415, 150 (2002).

[2] T.W. Hill, W.E. Wallace, R.S. Craig, T. Inoue, J. Solid State Chem. 8, 364 (1973).

[3] S. Barbara, M.F. Rossignol, J.X. Boucherle, Phys. Lett. A 55, 321 (1975).

[4] A.H. Millhouse, H.-G. Purwins, E. Walker, Solid State Commun. 11, 707 (1972).

[5] W. Schelp, A. Leson, W. Drewes, H.-G. Purwins, H. Grimm, Z. Phys. B 51, 41 (1983).

[6] T. Hashimoto, T. Kuzuhara, M. Sahashi, K. Inomata, A. Tomokiyo, W. Yayama, J. Appl. Phys. 62, 3873 (1987).

[7] P.J. von Ranke, N.A. de Oliveira, M.V. Tovar Costa E.P. Nobrega, A. Caldas, I.G. de Oliveira, J. Magn. Magn. Mater. 226-230, 970 (2001)

[8] J.C.P. Campoy, E.J.R. Plaza, A.A. Coelho, S. Gama, Phys. Rev. B 74, 134410 (2006).

[9] M.E. Wood, W.H. Potter, Cryogenics 25, 667 (1985).

[10] H. Oesterreicher, F.T. Parker, J. Appl. Phys. 55, 4334 (1984).

[11] M. Patra, S. Majumdar, S. Giri, Y. Xiao, T. Chatterji, J. Phys. Condens. Matter 26, 046004 (2014).

[12] I.G. de Oliveira, D.C. Garcia, P.J. von Ranke, J. Appl. Phys. 102, 073907 (2007). 